DIETER GRIMM

\title{
Gemeinwohl in der Rechtsprechung des Bundesverfassungsgerichts
}

\section{Der veränderte Gemeinwohlbegriff der Moderne}

Der Begriff des Gemeinwohls spielt in der Verfassungsrechtsprechung keine herausragende Rolle. Die Fundstellen in der hundertbändigen Entscheidungssammlung bleiben klein an Zahl. Nichts anderes gilt für die Verfassung selbst. Auch sie verwendet das Wort selten und fernab ihrer Zentralbegriffe. Daraus darf freilich nicht geschlossen werden, daß zwischen Verfassung und Gemeinwohl keine Beziehung bestünde. Es ist vielmehr aufschlußreich, daß die Verfassung in eben dem historischen Moment aufkommt, in dem das Gemeinwohl seine Eigenschaft als substantielle und feststehende Größe einbüßt. Dabei handelt es sich nicht bloß um eine zeitliche Koinzidenz. Die Entstehung der Verfassung und der Wandel des Gemeinwohlbegriffs sind vielmehr Produkt ein und desselben Prozesses.

Dieser Prozeß war ursprünglich in individualistischen Kategorien begründet worden, nämlich als Befreiung des Einzelnen aus der Bevormundung durch Staat und Kirche sowie aus den ständisch-feudalen Bindungen. Sie wichen den Prinzipien individueller Freiheit und Gleichheit mit der Folge der Umstellung der Rechtsordnung von objektiven Pflichten auf subjektive Rechte und der Reduzierung der Staatszwecke auf die Sicherung gleicher Freiheit. Er läßt sich aber auch überindividuell verstehen als Trennung von Staat und Gesellschaft, Ersetzung staatlicher Lenkung durch gesellschaftliche Selbststeuerung, Übergang von stratifikatorischer zu funktionaler Differenzierung oder Umstellung der Legitimationsgrundlage politischer Herrschaft von Wahrheit auf Freiheit.

Die Individualfreiheit erschien dabei einmal eher als Mittel zur Steigerung der allgemeinen Wohlfahrt, einmal eher als Selbstzweck im Sinne der Bestimmung des Menschen, stets aber als Ausdruck der Überzeugung, daß das Gemeinwohl nicht mehr von staatlicher Bewirkung, sondern von individueller Entfaltung und, vermittelt durch sie, von der Autonomie der verschiedenen gesellschaftlichen Funktionssysteme zu erwarten sei. Das Gemeinwohl wurde dadurch als Grund der Vergesellschaftung und Zweck politischer Herrschaft nicht etwa obsolet. Es verwandelte sich aber von einer vorgegebenen materialen Größe in die Resultante eines gesellschaftlichen Prozesses, dessen 
störungsfreien Verlauf der Staat unter Verzicht auf eigene Steuerungsambitionen zu garantieren hatte.'

Damit entstand die von der Arbeitsgruppe „Gemeinwohl und Gemeinsinn“ mehrfach registrierte Situation, daß das Gemeinwohl einerseits als Kriterium für die Bewertung von Herrschaftsansprüchen und Interessentenforderungen unverzichtbar blieb, andererseits aber inhaltlich entleert und dauerhaft strittig wurde. ${ }^{2}$ Der Gemeinwohlbegriff krankt seitdem an Unterscheidungsschwäche. Er kann für verschiedene, selbst gegensätzliche Inhalte in Anspruch genommen werden. Er läßt sogar Konvergenzen mit Eigennutz zu. Daraus darf aber nicht der Schluß gezogen werden, daß Gemeinwohlformeln nunmehr gänzlich beliebig verwendbar seien. Es steht vielmehr durchaus in Frage, ob dem Begriff des Gemeinwohls durch den, ,semantischen Coup“ des Liberalismus der Gegenbegriff des Eigennutzens abhanden gekommen ist. ${ }^{3}$

Zwar sind individuelle Freiheit und Autonomie der gesellschaftlichen Funktionsbereiche selbst zu Gemeinwohlbelangen geworden. Man darf den eigenen Nutzen verfolgen und doch hoffen, damit zum Wohl aller beizutragen. Ebenso dürfen die gesellschaftlichen Subsysteme ihren je eigenen Rationalitätskriterien gehorchen, während sie denjenigen anderer Subsysteme mit Indifferenz begegnen, und können doch gerade dadurch ihren Beitrag zum Gesamtwohl steigern. Das geht ohne Sprengung des gesellschaftlichen Zusammenhalts und ohne Senkung des gemeinsamen Leistungsstandards aber nicht unbegrenzt. Grenzen ergeben sich vielmehr aus der Gleichheit der Freiheit und der wechselseitigen Interdependenz der Subsysteme. Nicht jedes eigennützige Verhalten ist zugleich gemeinwohlförderlich.

Das wird vollends deutlich, wenn man den öffentlichen Sektor in die Betrachtung einbezieht. Das liberale Sozialmodell macht ja den Staat nicht überflüssig. Es verändert lediglich seine Legitimationsgrundlage und begrenzt seine Aufgaben. Sie bestehen in der Herstellung und Sicherung jener Voraussetzungen, unter denen die gesellschaftliche Selbststeuerung zum Gemeinwohl führt. Ihre Erfüllung verlangt aber gerade die Koordinierung und Begrenzung von Freiheitssphären. Dazu ist Macht erforderlich, die einerseits unwiderstehlich, andererseits aber zweckgebunden sein muß. Das staatliche Gewaltmonopol wird daher erst jetzt durch die restlose Privatisierung der Gesellschaft vollendet, zugleich aber in den Dienst der Privatautonomie gestellt. ${ }^{4}$ Seine Verwendung zum Eigennutz der Machthaber wäre unter keinem Gesichtspunkt rechtfertigungsfähig.

Das Problem liegt also nicht in der Grenzenlosigkeit des Gemeinwohlbegriffs durch Verschmelzung mit dem Eigennutz, sondern in der Unbestimmtheit des Grenzverlaufs zwischen gemeinwohlförderlichem (oder -neutralem) und gemeinwohlabträglichem Eigennutz. Darüber bestehen legitimerweise unterschiedliche Ansichten, zwischen denen nicht unter Rekurs auf Wahrheit ausgewählt werden kann. Das Gemeinwohl ist insofern pluralisiert. Die Entscheidung zwischen den verschiedenen Auffassungen muß nach anderen Gesichtspunkten getroffen werden. Unter den Bedingungen gleicher Frei-

Grimm 1987, vor allem S. 13 ff.

Münkler/Bluhm 2001; Münkler/Fischer 1999; Münkler/Fischer 2002a; Münkler/Fischer 2002b, S. $10 \mathrm{f}$.

3 Münkler/Fischer 1999, S. 247.

4 Grimm 1987, S. 192 ff. 
heit kann das nur in einem politischen Meinungs- und Willensbildungsprozeß geschehen, in dem bestimmt wird, was bis zu einer anderslautenden Entscheidung als Gemeinwohl gilt. Da das Gemeinwohl als Resultante aus dem Prozeß hervorgeht, kann es diesem nicht zugleich als Maßstab dienen. Das Gemeinwohl ist insofern prozeduralisiert.

\section{Die Verfassung als Reaktion auf den Wandel des Gemeinwohls}

An dieser Stelle kommt die Verfassung ins Spiel. ${ }^{5}$ Es ist vor allem die nicht endgültig abschließbare Bestimmungsbedürftigkeit des Gemeinwohls, die zur Verfassung drängt. Sie macht Regeln über die Berechtigung zur Gemeinwohldefinition und über die Voraussetzungen ihrer Verbindlichkeit nötig. Deswegen hat es auch nichts mit Zufall zu tun, daß unter den älteren Bedingungen der Gemeinwohlgewißheit keine Verfassung entstand. Gründet sich ein Herrschaftssystem auf Wahrheit statt auf Freiheit, erscheinen solche Bestimmungen dysfunktional. Sie werden wie Hemmnisse der von Gott geoffenbarten oder von einer Elite erkannten Wahrheit empfunden. Daraus wird auch verständlich, daß alle Neoabsolutismen des zwanzigsten Jahrhunderts sich von verfassungsrechtlichen Bindungen ganz frei machten oder Verfassungen von vornherein nur zum Schein erließen oder sie jedenfalls im Konflikt mit substantiellen Gemeinwohlanforderungen hintansetzten.

Demgegenüber formuliert die Verfassung Bedingungen für die Bestimmung des Gemeinwohls unter der Prämisse, daß über seinen Inhalt und seine Ermittlung verschiedene Auffassungen miteinander konkurrieren, die nicht anhand von Wahrheitskriterien beurteilt werden können. Manche Verfassungen beschränken sich dabei auf formale Regelungen, indem sie lediglich die Organe einrichten, die an der Bestimmung und Durchsetzung des Gemeinwohls beteiligt sind, und ihnen das Verfahren vorschreiben, das sie dabei zu beachten haben. Schon in den ersten Verfassungen wurden den Formalregeln aber inhaltliche Prinzipien in Gestalt von Staatszielbestimmungen und Grundrechten an die Seite gestellt, die beachtet werden mußten, wenn das Gemeinwohl nicht verfehlt werden sollte. Heute sind solche Regeln allgemein üblich. Einige Verfassungen, deren Vorbild oft das Grundgesetz ist, gehen darin so weit, gänzlich Unverfügbares zu normieren wie beispielsweise die Menschenwürde, die Demokratie, den Rechtsstaat etc.

Ohne ein vorgegebenes materiales Gemeinwohl ist die Verfassung freilich selbst das Ergebnis eines gesellschaftlichen Meinungs- und Willensbildungsprozesses. Indem dieses Ergebnis aber in Rechtsnormen gegossen wird, erhält es eine Verbindlichkeit, die über den Kreis der an der Entscheidung Beteiligten hinausreicht, wird auf Dauer gestellt und beansprucht Vorrang gegenüber den von der Staatsgewalt ausgehenden Herrschaftsakten. Die Verfassung hat, bezogen auf das Gemeinwohl, also eine doppelte Funktion: Zum einen konkretisiert sie die inhaltsarm gewordene Gemeinwohlformel, indem sie einige Optionen ausschließt und andere verbindlich macht und auf diese Weise die Pluralisierung des Gemeinwohls wieder reduziert. Zum anderen reguliert sie die Prozeduralisie-

5 Näher Grimm 2002, S. 31 ff.; Grimm 1998, S. 10 ff.; vgl. auch Luhmann 2000, S. 213. 
rung, indem sie den Meinungs- und Willensbildungsprozeß nach Akteuren, Kompetenzen und Verfahren strukturiert und Bedingungen für die Geltung von politischen Entscheidungen formuliert, die die Gemeinwohl-Verträglichkeit verbessern sollen.

Die Verfassung schließt damit aber nicht etwa die Lücke, die der ältere Gemeinwohlbegriff hinterlassen hat. Sie versieht lediglich einzelne Belange mit einem Gemeinwohl-Siegel, während sie es anderen abspricht. So wird beispielsweise in Art. 5 GG die Pressefreiheit ins Gemeinwohl einbezogen, die Zensur aus ihm ausgeschlossen. Angriffskrieg und Todesstrafe können unter der Geltung des Grundgesetzes keine Gemeinwohl-Eigenschaft beanspruchen (Art. 26 und 102 GG), die Gleichberechtigung von Männern und Frauen wird in den Rang eines Gemeinwohl-Erfordernisses erhoben (Art. 3 Abs. 2 GG). Um dies zu erreichen, braucht die Verfassung das Wort „Gemeinwohl“ aber nicht ständig im Munde zu führen. Es liegt vielmehr in ihrer Funktion begründet, daß die einzelnen Vorschriften positive oder negative Gemeinwohl-Festlegungen treffen, die selber nicht mehr Thema, sondern Prämisse politischer Entscheidungen sind. ${ }^{6}$

Die Grundlagen für die laufend anfallenden Gemeinwohlentscheidungen und diese Entscheidungen selbst werden dadurch auseinandergezogen. Jene müssen von einem breiten Konsens getragen sein, damit diese auch von knappen Mehrheiten getroffen werden können und dennoch bei den Unterlegenen Anerkennung finden. Die Verfassung erlaubt also Stabilität im Wandel. Wechselnde Gemeinwohl-Projekte finden in den dauerhaften Prinzipien Halt. Die Gemeinwohl-Frage steht nicht bei jeder politischen Entscheidung umfassend zur Diskussion. Sie ist immer in den Rahmen von Vorentscheidungen verwiesen, die im laufenden Verfahren nicht verändert werden dürfen. Die Verfassung vermag auf diese Weise zwischen der Unverzichtbarkeit und der Unbestimmtheit des Gemeinwohlbegriffs zu vermitteln.

\section{Verfassung und Gemeinsinn}

Der Gemeinsinn, den die Arbeitsgruppe ebenfalls thematisiert, und zwar als moralische Ressource, die über den Rechtsgehorsam hinausgeht und Bürgerengagement im Gemeininteresse hervorbringt, ${ }^{7}$ liegt dagegen außerhalb des Zugriffs der Verfassung. Dafür gibt es zwei Gründe: Der eine besteht darin, daß die Verfassungsnormen an die öffentliche Gewalt adressiert sind, nicht an den Bürger. Das heißt nicht, daß sie keinen Bezug zum Bürger hätten. Aber der Bürger ist der Berechtigte, verpflichtet wird der Staat. Man kann sich zwar andere Verfassungen vorstellen, entfernt sich dann aber gerade von dem, was die moderne Errungenschaft der Verfassung ausmacht. Der zweite Grund besteht darin, daß die Verfassung zwar Raum für Gemeinsinn gibt, aber nicht zum Gemeinsinn verpflichten kann, denn dieser entzieht sich als ein Fall von innerer Einstellung der rechtlichen Anordnung. Die Verfassung setzt daher nicht auf Gemeinsinn, sondern versucht den gesellschaftlichen Zusammenhalt gerade unter der Voraussetzung von Eigeninteresse zu sichern.

\footnotetext{
Luhmann 2000, S. 86.

Münkler/Fischer 1999; Münkler/Fischer 2002b, S. 9 f.; Münkler/Fischer in diesem Band.
} 
Das gilt auch für die Träger öffentlicher Gewalt als Regelungsadressaten der Verfassung. Von ihnen wird Verfassungs- und Rechtstreue verlangt, nicht aber eine darüber hinausweisende Gesinnung, die sie zu einem rechtlich nicht geforderten Einsatz anhält. Gewaltenteilung, Kompetenzgrenzen, Verfahrensregelungen, Rechenschaftspflichten, Korruptionsverbote begrenzen vielmehr das Feld, in dem die Inhaber staatlicher Ämter ihre Eigeninteressen verfolgen oder Willkür üben können. Der Verzicht auf Selbstbegünstigung und Willkür wird den Akteuren im Verfassungsstaat also nicht als individuelle moralische Leistung abverlangt, sondern strukturell im System verbürgt und dadurch mit höherer Wahrscheinlichkeit versehen. ${ }^{8}$

Mit wachsenden gesellschaftlichen Desintegrationstendenzen scheint die verfassungsrechtliche Gemeinsinn-Abstinenz freilich unbefriedigend. Deswegen begegnet man gelegentlich Versuchen, Gemeinsinn mit Mitteln der Verfassung zu fördern. So beantragten einige ostdeutsche Abgeordnete im Zuge der Verfassungsreform nach der Wiedervereinigung, das Grundgesetz um den Satz ,Jeder Mensch ist zur Mitmenschlichkeit und Gemeinsinn aufgerufen" zu ergänzen. Indessen handelt es sich dabei um einen Satz ohne Verfassungseignung. ${ }^{9}$ Charakteristikum der modernen Verfassung ist, daß sie die öffentliche Gewalt umfassend verrechtlicht. Sie besteht daher aus Normen, die an die Staatsgewalt adressiert sind und ihre Einrichtung und Ausübung regeln. Diese Normen sind mit Verbindlichkeit ausgestattet und erlauben juristische Feststellungen darüber, ob die Staatsgewalt rechtmäßig oder rechtswidrig innegehabt und ausgeübt wird. Aus dieser Beschränkung bezieht die Verfassung ihre Wirkungskraft.

Folglich eignet sich nicht jeder Sollenssatz, so wünschenswert oder bestandswichtig er für die Gesellschaft auch sein mag, zur Aufnahme in die Verfassung. Dem Aufruf zur Mitmenschlichkeit und Gemeinsinn fehlen alle Eigenschaften eines Verfassungssatzes. Er ist nicht an die Staatsgewalt gerichtet. Nicht ihr wird aufgetragen, diese Werte zur Maxime politischer Entscheidungen zu machen. Er wendet sich vielmehr an die Einzelnen: Sie sollen mit Hilfe der Verfassung angehalten werden, sich in Tugenden zu üben. Die Formulierung läßt auch keinen Willen zur Rechtsgeltung erkennen, sie erschöpft sich in einem Appell. Wenn dieser befolgt wird, ist das erfreulich, wenn er mißachtet wird, ist es bedauerlich. Juristisch ist es gleichgültig. Gerade deswegen kann man sich aber nicht damit beruhigen, daß solche Verfassungssätze jedenfalls nicht schadeten. Sie untergraben den Geltungsanspruch der Verfassung und tragen langfristig zu ihrer Erosion bei.

Die andere, anziehungskräftigere Variante besteht in der Forderung, den Grundrechtskatalog des Grundgesetzes um Grundpflichten zu ergänzen. Sie ist dort besonders oft zu vernehmen, wo die Ursache der gesellschaftlichen Desintegrationstendenzen in der grundrechtlichen Freiheit gesucht wird. Es unterliegt freilich keinem Zweifel, daß gesellschaftlicher Zusammenhalt ohne bürgerliche Pflichten undenkbar ist. Deswegen trifft die Verfassung dafür Vorsorge. Sie gibt dem Staat die Mittel, den Einzelnen die im Gesamtinteresse notwendigen Pflichten aufzuerlegen. Das gilt auch in den grundrechtlich gesicherten Bereichen. Die Grundrechte garantieren keine absolute Freiheit, sondern sind mit Beschränkungsvorbehalten versehen. Daher stellt sich die Frage, was Grundpflichten dem hinzufügen.

8 Luhmann 2000, S. 84 f., $140 \mathrm{ff}$.

9 Grimm 2001, S. 82. 
Man kann sich der Antwort über einen Umweg nähern, indem man fragt, was der Sinn von Grundrechten im Unterschied zu gewöhnlichen Rechten ist. ${ }^{10}$ Dem modernen Staat steht im Interesse von Friedenswahrung und Gemeinwohlverwirklichung das Gewaltmonopol zu, dem auf Seiten der Einwohner die Gehorsamspflicht entspricht. Er hat die Befugnis, Recht zu setzen und es im Ungehorsamsfall mit Zwangsgewalt durchzusetzen. Soll das Gewaltmonopol mit dem Prinzip der Individualfreiheit vereinbar sein, dann muß der Einzelne mit Rechten gegenüber der Staatsgewalt ausgestattet werden, die dieser vorgehen und die Rechtsetzungsbefugnis sowie den Gewalteinsatz begrenzen. $\mathrm{Daß}$ sie der Staatsgewalt vorgehen und von ihr zu achten sind, macht sie zu Grundrechten. Grundrechte verringern auf diese Weise das Gefälle zwischen Staat und Bürger, ja erst die Existenz von Grundrechten macht aus Untertanen Bürger.

Für Grundpflichten besteht eine vergleichbare Notwendigkeit nicht. Der Staat kann den Einzelnen auch ohne sie alle Pflichten, die im Interesse des verfassungsrechtlich definierten Gemeinwohls rechtfertigungsfähig sind, auferlegen. Der Einzelne ist ihnen unterworfen. Der Staat benötigt ihm gegenüber kein Gegengewicht, weil er ohnehin das Übergewicht besitzt. Mit der Einführung von Grundpflichten in die Verfassung würde die Balance, die durch Grundrechte zwischen Staatsgewalt und Bürger hergestellt wird, zugunsten des Staates wieder aufgehoben. Deswegen sind Grundpflichten zur Herstellung von Gemeinsinn das falsche Mittel. Darin liegt kein Plädoyer für die Entbehrlichkeit von Gemeinsinn. Seine Quellen müssen allerdings außerhalb des Rechts gesucht werden. Das Recht kann allenfalls günstige Voraussetzungen für die Entwicklung von Gemeinsinn schaffen.

\section{Verfassungsrechtsprechung als normvermittelter Gemeinwohl-Beitrag}

Ohne die Klärung des Verhältnisses von Verfassung und Gemeinwohl ist das Verhältnis von Verfassungsrechtsprechung und Gemeinwohl nicht verständlich. Verfassungsrechtsprechung ist die Anwendung von Verfassungsrecht auf konkrete Streitfälle. Das Bundesverfassungsgericht hat es folglich nicht unmittelbar mit dem Gemeinwohl, sondern mit dem Gemeinwohl in Form seiner verfassungsrechtlichen Konkretisierungen zu tun. In der Verfassung sind die von der Gesellschaft akzeptierten Bedingungen für gemeinwohl-orientierte Entscheidungen festgelegt. Das Verfassungsgericht prüft, ob diese im konkreten Fall eingehalten wurden. Die Verfassung schneidet damit für das Verfassungsgericht einen unmittelbaren Durchgriff auf „das Gemeinwohl“ ab, genauso wie sie einen unmittelbaren Durchgriff auf „die Gerechtigkeit" abschneidet. "' Andernfalls würde es aus der Funktion der Verfassungsanwendung heraustreten und sich zum Verfassungsgeber aufschwingen, damit das Demokratieproblem verschärfend, das ohnehin mit Verfassungsgerichtsbarkeit verbunden ist.

${ }^{10}$ Götz/Hofmann 1983, dort auch meine Diskussionsbeiträge, S. 102, 105.

11 Vgl. Robbers 1980, S. 56; Uerpmann 1999, S. 200. 
Allerdings darf man sich die Determination der richterlichen Tätigkeit durch Verfassungsrechtssätze nicht zu eng vorstellen. Der Positivismus des neunzehnten und frühen zwanzigsten Jahrhunderts, der die Norm mit ihrem Text identifizierte und Grammatik und Logik für die einzig zulässigen Mittel zur Ermittlung des Sinns von Normtexten hielt, liegt hinter uns. Die Rechtsprechung des Bundesverfassungsgerichts zeichnet sich methodologisch dadurch aus, daß der Normtext als Ausdruck eines Zwecks verstanden wird, der sich in der sozialen Wirklichkeit Geltung verschaffen soll. Deswegen nimmt es zur Deutung der Norm angesichts entscheidungsbedürftiger Fälle einerseits Rückgriff auf den Zweck, der hinter der Norm steht, und andererseits Vorgriff auf den Ausschnitt der sozialen Wirklichkeit, auf den sich die Norm regelnd bezieht. Die Norm ist so auszulegen, daß der Zweck unter den jeweiligen Wirklichkeitsbedingungen optimal verwirklicht wird.

Die Norm geht dann nicht mehr in ihrem Text auf. Dieser ist zwar Ausgangs- und Endpunkt der Sinnermittlung. Interpretationen, die sich mit dem Wortlaut nicht vereinbaren lassen, sind dem Rechtsanwender nicht erlaubt. Zwischen den ersten Textbefund und die abschließende Textvereinbarkeitskontrolle schieben sich aber Interpretationsschritte, bei denen sich eine je nach Dichte des Normtextes mehr oder weniger große Optionsspanne öffnet. Dabei stellt die Zweckberücksichtigung den Gemeinwohlbezug her. Jeder Normzweck läßt sich bis zum Begriff des Gemeinwohls zurückverfolgen. Allerdings ist dieser selbst inhaltsarm. Erst die Verfassungsrechtssätze reichern ihn inhaltlich an. Deswegen vermag er die von der Rechtsanwendung geforderten Antworten auf konkrete Probleme nicht zu instruieren. Die Rechtsprechung macht dementsprechend regelmäßig vorher halt.

So geht das Bundesverfassungsgericht beispielsweise bei der Interpretation der Medienfreiheit in Art. 5 GG davon aus, daß diese im Unterschied zu den meisten anderen Grundrechten keine Freiheit im Interesse der Grundrechtsträger, also der Medienunternehmer oder Journalisten, sondern eine Freiheit im Interesse des Publikums ist. Sie dient einer höheren Freiheit, von der sie ihren Sinn empfängt: der freien individuellen und öffentlichen Meinungsbildung. ${ }^{12}$ Die Freiheit des einzelnen im Medienwesen Tätigen genügt unter diesen Umständen nicht zur Zweckerreichung. Das Medienwesen als Ganzes muß so beschaffen sein, daß es den verfassungsrechtlich geforderten Dienst erbringt. Da die Gefahr für die Freiheitlichkeit nicht nur vom Staat, sondern auch von den im Medienwesen Tätigen selbst ausgeht, wenn sie es für andere als publizistische Zwecke instrumentalisieren, kann das so verstandene Grundrecht Beschränkungen der Individualfreiheit im Interesse der Freiheitlichkeit des Systems rechtfertigen.

Das ist eine anspruchsvolle und ungewöhnliche Konstruktion. Sie wird daraus abgeleitet, daß das Grundrecht der freien Entfaltung der Persönlichkeit von umfassender und wahrheitsgemäßer Information abhängt und da 3 das Prinzip der Demokratie ohne freie Kommunikation nicht verwirklicht werden kann. Persönlichkeitsentfaltung und Demokratie sind durch das Grundgesetz zu Gemeinwohltatbeständen von hohem Rang erklärt worden. Aber der Rückgriff auf das Gemeinwohl könnte dem Interpretationsergebnis weder etwas hinzufügen noch es abändern. Sätze wie der, daß ,die Bindung an das

12 Ständige Rechtsprechung seit BVerfGE 57, 295 (320). 
Gemeinwohl [...] selbstverständliche Voraussetzung jeder verfassungsrechtlich gebundenen Gesetzgebung " sei, ${ }^{13}$ bleiben daher ohne konkrete Auswirkungen.

Sehr wohl könnte sich das Ergebnis der Rundfunkrechtsprechung aber durch alternative Deutungen der Rundfunkfreiheit ändern, etwa dergestalt, daß der Zweck des Art. 5 GG allein in der Garantie der individuellen Freiheit der Mediatoren bestehe oder daß es zwar um die Freiheit des Medienwesens insgesamt gehe, diese aber durch den Markt hinreichend gesichert werde. Auch die Weichenstellungen für solche Interpretationen, die in Auseinandersetzung mit dem Bundesverfassungsgericht durchaus vertreten werden, fallen aber auf einer Konkretisierungsstufe, die weit unterhalb des Gemeinwohls liegt. Würde statt dessen zur Begründung des Interpretationsergebnisses kurzerhand auf das Gemeinwohl verwiesen, läge darin gerade eine Verschleierung der bestimmenden Interpretationsfaktoren.

\section{Gemeinwohlformeln im Grundgesetz}

Allerdings enthält das Grundgesetz einige Vorschriften, mit denen es selber den Durchgriff auf das Gemeinwohl nahe legt, indem es dieses zum Tatbestandsmerkmal macht. Es sind im gesamten Verfassungstext freilich nicht mehr als drei, denen man noch eine aus dem Bundesverfassungsgerichtsgesetz hinzugesellen kann. Bei näherer Betrachtung ändern aber auch diese Normen an dem Grundverhältnis von Verfassungsrechtsprechung und Gemeinwohl nichts. Sie bleiben entweder deklamatorisch oder weisen die Konkretisierung des Gemeinwohl-Tatbestandes dem Gesetzgeber zu. Das Bundesverfassungsgericht ist darauf beschränkt zu überprüfen, ob bei der Konkretisierung die verfassungsrechtlichen Anforderungen an gesetzgeberisches Handeln beachtet worden sind. Eigenständige Gemeinwohl-Definitionen trifft es auch hier nicht. ${ }^{14}$

Deklamatorische Funktion hat Art. 56 GG. Er formuliert den Amtseid des Bundespräsidenten, der gemäß Art. $64 \mathrm{GG}$ auch vom Bundeskanzler und den Bundesministern zu leisten ist. Alle schwören, ihre Kraft dem „Wohle des Deutschen Volkes“ widmen zu wollen. Angesichts des pluralisierten Gemeinwohls geht damit allerdings keine Verpflichtung auf bestimmte Inhalte einher. Der Amtsträger darf seiner Vorstellung vom Gemeinwohl folgen. Ausgeschlossen wird nur, daß er die Amtsgewalt zum persönlichen Wohl nutzt.

Die Vorschriften sind auch nicht mit Sanktionen verknüpft. Das Mißtrauensvotum gegen den Bundeskanzler ist ohne Bezug zum Amtseid. Hinsichtlich des Bundespräsidenten erlaubt Art. $61 \mathrm{GG}$ zwar die Präsidentenanklage, aber nicht wegen GemeinwohlVerfehlung, sondern wegen vorsätzlicher Verletzung des Grundgesetzes oder anderer Bundesgesetze. Der gern erhobene Vorwurf, ein Amtsträger habe seinen Eid gebrochen, denn seine Entscheidungen seien dem Gemeinwohl abträglich, ist ohne verfassungsrechtliche Grundlage. Hinter ihm stehen in der Regel lediglich divergierende Vorstel-

13 BVerfGE 50, 50 (51).

14 Deutlich BVerfGE 24, 367 (403 f.). Ausführliche Nachweise über die Verwendung des Begriffs in der Verfassungsjudikatur (bis 1969) bei Häberle $1970 \mathrm{a}$; vgl. weiter Häberle $1970 \mathrm{~b}$. 
lungen vom Gemeinwohl. Der Mißbrauch der amtlichen Stellung zum persönlichen Vorteil wird durch Normen sanktioniert, die nicht an Art. 56 und 64 GG anknüpfen.

Die zweite Verfassungsnorm, die unmittelbar auf das Gemeinwohl Bezug nimmt, ist die Eigentumsfreiheit des Art. 14 GG. Sein Absatz 2 lautet: „Eigentum verpflichtet. Sein Gebrauch soll zugleich zum Wohle der Allgemeinheit dienen." Absatz 3 läßt Enteignungen nur „zum Wohle der Allgemeinheit" zu. Entschädigungen sind unter gerechter $\mathrm{Ab}$ wägung "der Interessen der Allgemeinheit und der Beteiligten“ zu bestimmen. Beide bestätigen den bisherigen Befund. Art. 14 Abs. 2 GG enthält, da die Grundrechte Individuen berechtigen und den Staat verpflichten, keine Verpflichtung der Eigentümer, sondern nur eine Richtlinie für den Gesetzgeber. Er gibt ihm - im Verein mit Art. 14 Abs. 1 S. 2 (,Inhalt und Schranken werden durch die Gesetze bestimmt.“) - hinsichtlich des Eigentums weiterreichende Befugnisse, als bei den anderen Grundrechten vorgesehen.

Das Bundesverfassungsgericht prüft traditionell nur nach, ob der Gesetzgeber die Eigentumsfreiheit, die ja ihrerseits ein Gemeinwohl-Belang ist, übermäßig beschränkt hat. Zwar wirken die Grundrechte nicht nur als Grenzen des Staates im Freiheitsinteresse ihrer Träger, sondern nach der Rechtsprechung des Bundesverfassungsgerichts auch als Schutzpflichten des Staates gegenüber Freiheitsgefahren von dritter Seite. ${ }^{15}$ Daraus hat das Bundesverfassungsgericht Gesetzgebungsaufträge abgeleitet. Gesetze können danach nicht nur verfassungswidrig sein, weil sie in der Beschränkung einer grundrechtlichen Freiheit zu weit gehen, sondern auch weil sie zum Schutz einer gesellschaftlich bedrohten Freiheit zu wenig tun. Dem Übermaßverbot beim Eingriff entspricht ein Untermaßverbot bei der Schutzpflicht.

Es gibt aber bisher keinen Fall, in dem gerade unter Berufung auf Art. 14 Abs. 2 GG vom Gesetzgeber weitergehende Beschränkungen verlangt worden wären. Das könnte sich freilich ändern, falls die gegenwärtigen neoliberalen Bestrebungen zur Aufhebung bestehender Eigentumsbeschränkungen führen sollten. Auch dann ist aber nicht damit zu rechnen, daß solche Gesetzgebungsaufträge unmittelbar auf den Gemeinwohltatbestand in Art. 14 Abs. 2 GG gestützt werden. Vielmehr müßte dargelegt werden, welchen konkreten Verfassungsrechtsgütern vom Gebrauch der Eigentumsfreiheit Gefahren drohen, die vom Gesetzgeber zu bekämpfen sind.

Art. 14 Abs. 3 GG will mit dem Verweis auf das „Wohl der Allgemeinheit“ ausschließen, daß ein Privateigentümer sein Eigentum dem privaten Nutzen eines anderen aufopfern muß. Das Eigentumsopfer wird dem Einzelnen nur im Interesse der Allgemeinheit zugemutet. Regelmäßig geht der enteignete Gegenstand daher in öffentliches Eigentum über. Doch ist eine Enteignung zugunsten Privater nicht ausgeschlossen, sofern diese den Gegenstand - zumindest auch - im öffentlichen Interesse (etwa zur Schaffung von Arbeitsplätzen) verwenden. Im Zusammenhang mit der Entschädigung durchbricht die Gemeinwohlformel das Prinzip des vollen Wertausgleichs.

Die dritte Bezugnahme auf das Gemeinwohl enthält der später hinzugekommene Art. 87e Abs. 4 GG. Er steht im Zusammenhang mit der Privatisierung der Bundesbahn. Die Privatisierung hat zur Folge, daß die Bahn von dem Status grundrechtlicher Bindung in denjenigen grundrechtlicher Freiheit überwechselt. Sie kann ihr Verhalten folglich an wirtschaftlichen Kriterien ausrichten. Angesichts der damit verbundenen Gefahr für die

${ }^{15}$ Seit BVerfGE 39, 1 (4l ff.). 
Nutzer der Dienstleistung hat der Bund zu gewährleisten, „daß dem Wohl der Allgemeinheit [...] Rechnung getragen wird“" Was darunter zu verstehen ist, wird sogleich beispielhaft genannt: Die Verkehrsbedürfnisse des Publikums müssen beim Ausbau und Erhalt des Schienennetzes und bei den Verkehrsangeboten berücksichtigt werden. Wie das zu geschehen hat, bestimmt aber auch hier der Gesetzgeber, dessen Regelungen sich an den genannten oder ähnlichen Gemeinwohlbelangen legitimieren müssen.

Schließlich darf das Bundesverfassungsgericht nach $\S 32$ BVerfGG einstweilige Anordnungen erlassen, wenn dies ,zum gemeinen Wohl“ dringend geboten ist. Das Gericht bedient sich bei der Anwendung dieser Vorschrift einer Folgenabwägung - zum einen für den Fall, daß die einstweilige Anordnung ergeht, die Klage in der Hauptsache aber keinen Erfolg hat, zum anderen für den Fall, daß die einstweilige Anordnung verweigert wird, die Klage in der Hauptsache aber Erfolg hat. Die Folgen sind erwartbare Wirkungen im tatsächlichen Bereich. Sie müssen unter dem rechtlichen Gesichtspunkt des Gemeinwohls bewertet werden. Das Gemeinwohl wird aber auch hier klein gearbeitet, indem nach den Folgen für die betroffenen, zum Gemeinwohl zählenden konkreten Rechtsgüter gefragt wird, während das Gemeinwohl selbst nur noch in stereotyper Wiederholung der Gesetzesformulierung auftaucht.

\section{Gemeinwohl-Argumente in der Verfassungsrechtsprechung}

Die Hauptfundstelle für Gemeinwohlargumente bilden allerdings nicht Entscheidungen zu denjenigen Verfassungsnormen, in denen der Begriff selbst vorkommt. Am häufigsten trifft man sie vielmehr im Zusammenhang mit dem Prinzip der Verhältnismäßigkeit. Dabei handelt es sich um eine richterliche Rechtsschöpfung, die den Grundrechtsschutz wesentlich verstärkt. Das Prinzip verlangt, „daß eine Grundrechtsbeschränkung von hinreichenden Gründen des Gemeinwohls gerechtfertigt ist, das gewählte Mittel zur Erreichung des Zwecks geeignet und erforderlich ist und bei einer Gesamtabwägung zwischen der Schwere des Eingriffs und dem Gewicht der ihn rechtfertigenden Gründe die Grenze des Zumutbaren noch gewahrt ist" “. ${ }^{16}$

Die Rechtsprechung beginnt mit der Leitentscheidung zum Grundrecht der Berufsfreiheit aus Art. $12 \mathrm{GG}$, dem sogenannten Apotheken-Urteil. ${ }^{17}$ Das Bundesverfassungsgericht begegnete hier einem Verfassungstext, der die Freiheit der Berufswahl garantiert, für die Berufsausübung aber gesetzliche Regelungen zuläßt. Bei seiner Auslegung wurde jedoch schnell klar, daß regelungsbedürftig auch die Berufswahl sein kann, etwa wenn es um diejenigen Voraussetzungen geht, unter denen man bestimmte Berufe aufnehmen darf. Das Bundesverfassungsgericht interpretierte Art. 12 GG daher als einheitliches Recht der Berufsfreiheit, bei dem Wahl und Ausübung lediglich Pole einer gleitenden Skala sind. Es brachte die im Grundgesetz getroffene Unterscheidung aber zur Geltung, indem es verschieden hohe Anforderungen an berufsregelnde Gesetze stellt, je nachdem ob die Regelung näher an dem Pol der Wahl oder dem der Ausübung liegt. Für die Abstufung sind Gemeinwohl-Erwägungen ausschlaggebend.

\footnotetext{
16 BVerfGE 78, 77 (85).

17 BVerfGE 7, 377.
} 
Das Verfassungsgericht ging davon aus, daß die Grundrechte die Individualfreiheit schützen sollen, während der Regelungsvorbehalt den Schutz von Gemeinschaftsinteressen sicherstellt. ${ }^{18}$ Die Freiheit der Berufsausübung ist unter diesen Umständen schon beschränkbar, wenn ,,vernünftige Erwägungen des Gemeinwohls es zweckmäßig erscheinen lassen“. Die Freiheit der Berufswahl darf dagegen nur eingeschränkt werden, „soweit der Schutz besonders wichtiger Gemeinschaftsgüter es zwingend erfordert". Die höchsten Anforderungen werden bei den sogenannten objektiven Zulassungsschranken, auf deren Erfüllung der Einzelne keinen Einfluß hat, errichtet. Sie sind nur zur Abwehr „nachweisbarer oder höchstwahrscheinlicher schwerer Gefahren für ein überragend wichtiges Gemeinschaftsgut" statthaft. ${ }^{19}$ Diese „Stufenlehre“ wird seitdem formelhaft wiederholt, so daß der Gemeinwohlbegriff im Zusammenhang mit Art. 12 GG am weitaus häufigsten auftaucht.

Regelmäßig wird der Gemeinwohlbegriff ferner bei dem aus dem Rechtsstaatsprinzip abgeleiteten Verbot rückwirkender Gesetze verwendet. Das Verfassungsgericht unterscheidet dabei zwischen echter und unechter Rückwirkung. Die unechte Rückwirkung, bei der ein in der Vergangenheit begründeter Sachverhalt mit Wirkung für die Zukunft anders geregelt wird, ist erlaubt, wenn bei der Abwägung das Wohl der Allgemeinheit das Vertrauen der Betroffenen in den Bestand des Gesetzes überwiegt. Echte Rückwirkung, die die Wirkungen der Neuregelung auch in die Vergangenheit erstreckt, ist nur ausnahmsweise zulässig, wenn ,zwingende Gründe des gemeinen Wohls“ auf dem Spiel stehen. ${ }^{20}$ Auch hier taucht der Gemeinwohlbegriff also im Zusammenhang mit dem Abwägungsprinzip auf, das seinerseits nur noch selten unter Rückgriff auf das Gemeinwohl formuliert wird.

Bisweilen heißt es, daß Grundrechtsbeschränkungen von „hinreichenden Gründen des Gemeinwohls" gerechtfertigt sein müssen. ${ }^{21}$ Das Gemeinwohl bezieht sich hier also auf den Gesetzeszweck, der hinter der grundrechtsbeschränkenden Regelung steht. Normalerweise ist aber nur von der Notwendigkeit eines legitimen Zwecks die Rede. Gemeint ist damit ein Zweck, dessen Verfolgung die Verfassung dem Gesetzgeber nicht untersagt. Die endgültige Entscheidung fällt gewöhnlich bei der Abwägung zwischen der Bedeutung des beschränkten Grundrechts und der Intensität der Beschränkung einerseits sowie der Bedeutung des hinter dem Gesetz stehenden Gemeinwohlzwecks und dem Grad seiner Gefährdung durch den Grundrechtsgebrauch andererseits.

Daran zeigt sich, daß die Entgegensetzung von Individualfreiheit und Gemeinwohl im Apotheken-Urteil mißverständlich war. Die Freiheit ist ebenso ein Gemeinwohlbelang, wie der legitimierende Grund für Freiheitsbeschränkungen einer ist, so daß in der Regel nicht Partikularwohl gegen Gemeinwohl steht, sondern konkurrierende Gemeinwohlbelange ins Verhältnis zu setzen sind. Später wird das auch ausdrücklich anerkannt. In einer Entscheidung aus dem Jahr 1976 führte das Bundesverfassungsgericht aus, das Grundgesetz habe nicht eine ,virtuell allumfassende Staatsgewalt verfaßt, son-

${ }^{8}$ BVerfGE 7, 377 (404)

${ }^{19}$ BVerfGE 7, 377 (378).

${ }^{20}$ BVerfGE 72, 200 (258).

${ }^{21}$ BVerfGE 78, $77(85)$. 
dern den Zweck des Staates materialiter auf die Wahrung des Gemeinwohls beschränkt, in dessen Mitte Freiheit und soziale Gerechtigkeit stehen". ${ }^{22}$

Damit eine Abwägung vorgenommen werden kann, muß das Gemeinwohl klein gearbeitet werden. Steht Gemeinwohl undifferenziert gegen Gemeinwohl, ist keine Abwägung möglich. Das Gemeinwohl wird also nur in der konkretisierten Form spezifischer Rechtsgüter relevant. Das können sowohl Grundrechte anderer wie andere Grundrechte, aber auch wichtige Gemeinschaftsgüter sein, die Volksgesundheit, die Verteidigungsfähigkeit, die Funktionsfähigkeit der Strafrechtspflege etc. Freilich ist gegenüber derartigen Großgütern des Gemeinwohls Vorsicht geboten, weil sie als solche selten auf dem Spiel stehen, wenn unkonkretisiert in die Waagschale gelegt, aber Abwägungsergebnisse zu Lasten der Freiheit präjudizieren.

Undifferenziert ist der Gemeinwohlbegriff nur als allgemeinster Zurechnungspunkt für die Rechtfertigung von Grundrechtsbeschränkungen brauchbar. Bei Fallentscheidungen ist der konkrete, durch das Grundgesetz mit einem Gemeinwohlsiegel versehene Rechtfertigungsgrund ausschlaggebend. Man kann daher mit Schuppert ${ }^{23}$ vom Bundesverfassungsgericht als Hüter des Gemeinwohls nur sprechen, wenn man darunter keine verfassungsunabhängige Gemeinwohlpflege versteht. $\mathrm{Zu}$ einer solchen ist das Gericht nicht befugt. Wenn es sie übernähme, würde es den im Grundgesetz verbindlich gemachten Gemeinwohlkonsens gerade durchkreuzen und der Gesellschaft seine eigenen Gemeinwohlvorstellungen aufoktroyieren, die nicht aus demjenigen Verfahren hervorgegangen sind, das dafür vorgesehen ist.

\section{Gemeinwohl durch die Rechtsprechung des Bundesverfassungsgerichts}

Der Gemeinwohlbeitrag des Bundesverfassungsgerichts besteht darin, daß es den in der Verfassung niedergelegten Gemeinwohl-Belangen und -Voraussetzungen im Konfliktfall Beachtung verschafft. Die Anlässe dafür sind in einem relativ gefestigten Verfassungsstaat wie der Bundesrepublik seltener absichtsvolle Mißachtungen der verfassungsrechtlichen Gemeinwohl-Anforderungen. Dagegen treten oft Meinungsverschiedenheiten darüber auf, was der Sinn einer verfassungsrechtlichen Regelung angesichts eines konkreten Problems ist. Der hohe Abstraktionsgrad zahlreicher Verfassungsnormen und die Notwendigkeit, sie auch auf Probleme zu beziehen, die zur Entstehungszeit der Verfassung nicht bedacht worden oder nicht vorhersehbar gewesen waren, erleichtert solche Differenzen. Verfassungskonflikte, wiewohl rhetorisch oft mit dem Vorwurf des Verfassungsverstoßes ausgetragen, sind daher nicht notwendig ein Indikator für mangelnden Verfassungsrespekt der Politik.

Zum Gemeinwohl-Problem werden solche Meinungsverschiedenheiten erst durch das Fehlen einer Konfliktlösungsinstanz. Die Mehrheit kann dann regelmäßig ihre Auffassung durchsetzen. Darunter leidet die machtbegrenzende Funktion der Verfassung

${ }^{22}$ BVerfGE 42, 312 (332).

23 Schuppert 2002. 
ebenso wie die zivilisierende Wirkung des Richtungsgrenzen überbrückenden Verfassungskonsenses. Diese Gefahr wächst mit der zunehmenden Professionalisierung der Politik. Sie hat ihre Wurzel in dem Umstand, daß in einer funktional differenzierten Gesellschaft die verschiedenen Funktionsbereiche spezifischen Rationalitätskriterien folgen, die gerade für sie bestimmend wirken. Für die Politik liegt dieses Rationalitätskriterium in Konkurrenzdemokratien in dem Imperativ des Machtgewinns und Machterhalts. Sie sind Voraussetzungen dafür, daß eine Partei ihren Vorstellungen vom Gemeinwohl mit Hilfe der Staatsgewalt Allgemeinverbindlichkeit verleihen kann. Macht vermittelt im demokratischen Verfassungsstaat die Wahl. Wahlgewinn ist daher das vordringliche Ziel der politischen Parteien. Aus ihrer Sicht erscheint es folglich rational, alles zu tun, was den Wahlerfolg fördert, und alles zu meiden, was ihn gefährdet.

Die gebieterische Wirkung dieses Codes trägt nicht allein die Orientierung am kurzfristigen Nutzen für die politischen Parteien in die Politik, während die langfristigen Folgekosten angesichts der stets bevorstehenden Wahl vernachlässigt werden. Sie führt auch dazu, daß andere an die Politik adressierte Verhaltensmaßstäbe wie die in der Verfassung niedergelegten Gemeinwohl-Bedingungen jedenfalls nicht den primären Orientierungspunkt politischen Handelns bilden. Sobald sie mit den Macherhaltungsinteressen der politischen Parteien in Konflikt geraten, werden sie als lästige Restriktionen wahrgenommen. Daraus folgt selbst für eine Politik, die ihre Verfassungsbindung nicht in Frage stellt, die Neigung, diese Restriktionen unter dem Gesichtspunkt der eigenen politischen Absichten zu interpretieren und sich zugunsten ihrer Eigeninteressen auch darüber hinwegzusetzen, wenn der Erfolg gemessen am politischen Code größer zu sein verspricht als der Nachteil.

Ohne ein Verfassungsgericht, das nicht der politischen Logik folgen muß, sondern sich frei von Rücksichten auf den Machtcode der Politik der Sinnermittlung von Verfassungsnormen widmen kann, fänden diese Tendenzen kein ausreichendes Gegengewicht. Demgegenüber sorgt schon die Existenz eines Verfassungsgerichts dafür, daß die Frage der Verfassungsmäßigkeit im politischen Entscheidungsprozeß relativ frühzeitig und relativ neutral thematisiert wird. Diese Vor-Wirkung der Verfassungsrechtsprechung ist daher unter Gemeinwohlgesichtspunkten nicht geringer einzuschätzen als die der politischen Entscheidung nachfolgende Kontrolltätigkeit, auf deren Existenz die Vor-Wirkung freilich beruht. Versagt diese, so sind bei Überschreitung des Handlungsrahmens, den die Verfassung der Politik zieht, Korrekturen möglich.

Die wichtigsten Anlässe für solche Korrekturen stammen heute aus den geschilderten Defiziten der Parteiendemokratie. Da diese strukturell bedingt sind und sich daher durch Appelle an die bessere Einsicht der Akteure nicht beheben lassen, ist Abhilfe nur von Institutionen zu erwarten, die in der Lage sind, die Eigenlogik des Parteibetriebs zu durchbrechen. Verfassungsgerichte sind mittlerweile die wichtigsten Institutionen dieser Art. Gegenüber der Kurzfristorientierung der Politik erinnern sie die parteipolitisch dominierten Staatsorgane an ihre verfassungsmäßig begründeten Langfristverpflichtungen. Sie schreiten dort ein, wo verfassungsrechtliche Handlungspflichten aus Furcht vor unpopulären Entscheidungen oder aus Rücksicht auf den Widerstand mächtiger gesellschaftlicher Interessengruppen unterbleiben. Sie verschaffen dem verfassungsrechtlichen Gleichheitspostulat Beachtung, wo sich die Parteien mittels einseitiger Begünsti- 
gungen aus staatlichen Ressourcen Gefolgschaft zu sichern versuchen. Vor allem werden sie dort begrenzend tätig, wo die staatlichen Ressourcen zur Selbstbegünstigung verwendet werden wie etwa bei der Parteienfinanzierung.

Neben dem Gemeinwohl in der Rechtsprechung des Bundesverfassungsgerichts steht deswegen das Gemeinwohl durch die Rechtsprechung des Bundesverfassungsgerichts. Dessen Referenzpunkte sind die in der Verfassung niedergelegten Gemeinwohlprinzipien. Diese sind allerdings vielfach so vage und lapidar formuliert, daß in den Prozeß der Verfassungsanwendung stets auch ein Quantum an Verfassungsschöpfung eingeht. Insofern bildet die Verfassungsgerichtsbarkeit selber ein Gemeinwohl-Problem. Denn angesichts des pluralisierten und prozeduralisierten Gemeinwohls ist es nicht Sache eines Gerichts, das aus guten Gründen nicht in den demokratischen Verantwortungszusammenhang einbezogen ist, genuine Gemeinwohl-Entscheidungen zu treffen. Allerdings besitzt auch die Verfassungsgerichtsbarkeit ein institutionelles Eigeninteresse an der Erhaltung ihrer Funktionsvoraussetzungen. $\mathrm{Zu}$ diesen gehört es, daß sie in ihrer Rechtsprechung plausibel machen kann, ihre Entscheidungen aus der Verfassung abgeleitet $\mathrm{zu}$ haben und nicht etwa subjektiven Präferenzen oder gar den Wünschen der Parteien gefolgt zu sein. ${ }^{24}$ Die Verselbständigung des Verfassungsgerichts findet darin ebenso eine Grenze wie in dem Recht der Politik, seine Rechtsprechung durch Änderungen der Verfassung anders zu programmieren.

\section{Literatur:}

Götz, V./Hofmann, H. (1983), Grundpflichten als verfassungsrechtliche Dimension, in: Veröffentlichungen der Vereinigung der deutschen Staatsrechtslehrer, Bd. 41, Berlin.

Grimm, D. (1987), Recht und Staat der bürgerlichen Gesellschaft, Frankfurt/M.

Grimm, D. (1998), Deutsche Verfassungsgeschichte 1776-1866. Vom Beginn des modernen Verfassungsstaats bis zur Auflösung des Deutschen Bundes, Frankfurt/M.. ${ }^{3}$.

Grimm, D. (2000), Constitutional Adjudication and Democracy, in: Judicial Review in International Perspective. Liber Amicorum in Honour of Lord Slynn of Hadley, hg. v. M. Adenas, Den Haag, S. 103.

Grimm, D. (2001), Die Verfassung und die Politik. Einsprüche in Störfällen, München.

Grimm, D. (2002), Die Zukunft der Verfassung, Frankfurt/M. ${ }^{3}$.

Häberle, P. (1970a), „Gemeinwohljudikatur" und Bundesverfassungsgericht. Öffentliche Interessen, Wohl der Allgemeinheit in der Rechtsprechung des BVerfG, in: Archiv des Öffentlichen Rechts, Bd. 95 , S. 86-125, 260-298.

Häberle, P. (1970b), Öffentliches Interesse als juristisches Problem, Bad Homburg.

Luhmann, N. (2000), Die Politik der Gesellschaft, Frankfurt/M.

Münkler, H./Bluhm, H. (Hg., 2001), Gemeinwohl und Gemeinsinn. Historische Semantiken politischer Leitbegriffe, Berlin.

Münkler, H./Fischer, K. (1999), Gemeinwohl und Gemeinsinn. Thematisierung und Verbrauch soziomoralischer Ressourcen in der modernen Gesellschaft, in: Berlin-Brandenburgische Akademie der Wissenschaften: Berichte und Abhandlungen, Bd. 7, Berlin, S. 237-265.

Münkler, H./Fischer, K. (Hg., 2002a), Gemeinwohl und Gemeinsinn. Rhetoriken und Perspektiven sozial-moralischer Orientierung, Berlin.

${ }^{24}$ Grimm 2000 . 
Münkler, H./Fischer, K. (2002b), Einleitung: Rhetoriken des Gemeinwohls und Probleme des Gemeinsinns, in: Münkler/Fischer 2002a, S. 9-17.

Robbers, G. (1980), Gerechtigkeit als Rechtsprinzip - Über den Begriff der Gerechtigkeit in der Rechtsprechung des Bundesverfassungsgerichts, Baden-Baden.

Schuppert, G. F. (2002), Gemeinwohl, das. Oder: Über die Schwierigkeiten, dem Gemeinwohlbegriff Konturen zu verleihen, in: Das Gemeinwohl: Auf der Suche nach Substanz. WZB-Jahrbuch 2002, hg. v. G. F. Schuppert/F. Neidhardt, Berlin (i. E.).

Uerpmann, R. (1999): Das öffentliche Interesse. Seine Bedeutung als Tatbestandsmerkmal und als dogmatischer Begriff, Tübingen. 